\title{
A NOTE ON COMPACT IDEAL PERTURBATIONS IN SEMIFINITE VON NEUMANN ALGEBRAS
}

\author{
FLORIN POP
}

(Communicated by Palle E. T. Jorgensen)

\begin{abstract}
Let $M$ be a semifinite von Neumann algebra and denote by $J(M)$ the closed two-sided ideal generated by the finite projections in $M$. A subspace $S \subset M$ is called local if it is equal to the ultraweak closure of $S \cap J(M)$. If $M=B(H)$ and $J(M)=K(H)$, Fall, Arveson, and Muhly proved that $S+J(M)$ is closed for every local subspace $S$.

In this note we prove that if $M$ is a type $\mathrm{II}_{\infty}$ factor, then there exist local subspaces in $M$ which fail to have closed compact ideal perturbations; thus answering in the negative a question of Kaftal, Larson, and Weiss.
\end{abstract}

\section{INTRODUCTION}

It is well known that if $I$ is a closed two-sided ideal in a $C^{*}$-algebra $A$ and if $B$ is a $C^{*}$-subalgebra of $A$ then $B+I$ is closed [3]. The proof relies on the fact that a one-to-one ${ }^{*}$-homomorphism between two $C^{*}$-algebras is automatically isometric.

This is no longer the case if we drop the assumption that $B$ is selfadjoint. However, if $A=B(H)$, the algebra of bounded operators on a Hilbert space $H$, and if $I=K(H)$, the ideal of compact operators, certain classes of (nonselfadjoint) algebras are known to have closed compact perturbations.

Attention has been especially devoted to the class of reflexive algebras. If $L \subset B(H)$ is a strongly closed lattice of projections containing 0 and $I$, define

$$
\operatorname{Alg} L=\{x \in B(H) ;(I-p) x p=0 \text { for every } p \in L\},
$$

and, dually, if $A$ is a subalgebra of $B(H)$, define

$$
\text { Lat } A=\{p \in B(H) \text { projection; }(I-p) x p=0 \text { for every } x \in A\} \text {. }
$$

A subalgebra $A \subset B(H)$ is said to be reflexive if $A=\operatorname{Alg}$ Lat $A$.

It is known that nest algebras (i.e., reflexive algebras with Lat $A$ totally ordered) have closed compact perturbations [4] and also certain classes of CSLalgebras (i.e., reflexive algebras with Lat $A$ commutative) (see $[6,9]$ ).

The strongest result in this direction is due to Fall, Arveson, and Muhly [4] and states that if $S$ is equal to the ultraweak closure of $S \cap K(H)$, then $S+K(H)$ is closed. Such subspaces were called local [4]. The proof heavily

Received by the editors March 10, 1992.

1991 Mathematics Subject Classification. Primary 46L10, 46H10. 
used the dualities $K(H)^{*}=C^{1}(H)$ and $C^{1}(H)^{*}=B(H) .\left(C^{1}(H)\right.$ is the ideal of trace-class operators on $H$.)

Notably, all the examples of CSL-algebras previously mentioned were proved to be local. Several examples of reflexive algebras are known to have nonclosed compact perturbations $[1,5]$, but all of them were shown to contain no nonzero compact operators. Let us also mention here the further deep results of Davidson and Power on perturbations and $M$-ideals in $C^{*}$-algebras [2].

If, more generally than $B(H)$, we consider a semifinite von Neumann algebra $M$, define $J(M)$ to be the norm-closed two-sided ideal generated by the finite projections in $M$. This ideal, called the compact ideal space of $M$, behaves in many ways like $K(H)$, but there also is notable difference, and completely new techniques are sought in this case (see, e.g., [10]).

If $L \subset M$ is a totally ordered strongly closed family of projections containing 0 and $I$ (i.e., a nest), then $M \cap \operatorname{Alg} L$ is called the nest-subalgebra of $M$ corresponding to $L$ [7].

Define a subspace $S \subset M$ to be local if $S$ equals the ultraweak closure of $S \cap J(M)$.

The first perturbation result in semifinite algebras was recently obtained by Kaftal, Larson, and Weiss [8], who proved that if $A \subset M$ is a nest-subalgebra, then $A+J(M)$ is norm-closed.

The major difficulty of the proof was that duality arguments were no longer available, since $J(M)^{* *} \neq M$; although they were able to show that nestsubalgebras of semifinite von Neumann algebras are local. The question whether a Fall-Arveson-Muhly-type theorem holds true arose naturally:

Problem [8]. If $M$ is semifinite and if $S \subset M$ is a local subspace, is $S+J(M)$ closed?

The purpose of this paper is to answer this question in the negative, thus leading to the conclusion that in the continuous semifinite case perturbation problems are more difficult than in the discrete case, and new techniques, which might differ from case to case, are required.

\section{A COUNTEREXAMPLE}

Let $M$ be a type $\mathrm{II}_{\infty}$ factor represented as $M_{0} \otimes B\left(l^{2}(\mathbb{N})\right)$ where $M_{0}$ is a type $\mathrm{II}_{1}$ factor with faithful normal trace $\tau$. Fix $\varepsilon>0$ and consider the operators

$$
x_{n}=q \oplus \underbrace{\varepsilon p_{1} \oplus \varepsilon p_{1} \oplus \cdots \oplus \varepsilon p_{1}}_{n \text { times }} \oplus p_{2} \oplus p_{3} \oplus \cdots,
$$

$x_{n} \in M, p_{i}$ projections in $M_{0} ; p_{1} \geq p_{2} \geq \cdots, \tau\left(p_{n}\right)=2^{-n}, \tau(q)>0$.

Define $S_{\varepsilon}$ to be the ultraweakly closed linear span of the operators $x_{n} . S_{\varepsilon}$ is local since $x_{n} \in J(M)$ for every $n \geq 1$.

Lemma 2.1. For every $x \in S_{\varepsilon} \cap J(M)$ there exist:

(i) an increasing sequence of integers $1 \leq n_{1}<n_{2}<\cdots<n_{k}<\cdots$ and

(ii) a convergence series $\sum_{k \geq 1} \lambda_{k}$ of complex numbers such that for every $k \geq 1$ there exists a net $\left(y_{\alpha}^{k}\right)_{\alpha \in A_{k}}$ of finite linear combinations of $x_{n}$ 's, $n>n_{k}$, such that the net

$$
x_{\alpha}^{k}=\lambda_{1} x_{n_{1}}+\cdots+\lambda_{k} x_{n_{k}}+y_{\alpha}^{k}
$$


converges to $x$ and $A_{k+1}$ is a cofinal subset of $A_{k}$.

Moreover, for every $\delta>0$ there is $k_{0}=k_{0}(\delta)$ such that, if $s_{\alpha}^{k}$ denotes the sum of the coefficients in $y_{\alpha}^{k}$, we have

$$
\begin{gathered}
\left|s_{\alpha}^{k}\right|<\delta \quad \text { for every } k \geq k_{0}, \\
\alpha \geq \alpha_{k} \in A_{k} \quad \text { for some } \alpha_{k} \in A_{k} .
\end{gathered}
$$

Proof. Let $\left(\xi_{n}\right)_{n \geq 0}$ be an orthonormal basis in $l^{2}(\mathbb{N})$, and let $E_{n}$ denote the projection onto the one-dimensional space generated by $\xi_{n}$. For any diagonal operator $x \in M \otimes B\left(l^{2}(\mathbb{N})\right),\left(I \otimes E_{n}\right) x$ will be referred to as the $n$th component of $x$.

If $x=0$, simply take $n_{k}=k, \lambda_{k}=0$; hence, let us assume that $x$ is different from 0 .

Since $x \in S_{\varepsilon}$, there exists a net of finite linear combinations of $x_{n}$ 's which converges ultraweakly to $x$. This net has the form

$$
\left(\mu_{1}^{\alpha} x_{1}+\mu_{2}^{\alpha} x_{2}+\cdots+\mu_{n}^{\alpha} x_{n}+\cdots\right)_{\alpha \in A}
$$

where all but finitely many $\mu_{n}^{\alpha}$ 's are equal to 0 . If $s_{\alpha}=\mu_{1}^{\alpha}+\mu_{2}^{\alpha}+\cdots+\mu_{n}^{\alpha}+\cdots$, the zeroth component of this net shows that $\lim _{\alpha} s_{\alpha}=s$ for some $s \in \mathbb{C}$.

If $m_{\alpha}=\min \left\{i ; \mu_{i}^{\alpha} \neq 0\right\}$, we claim that $\left(m_{\alpha}\right)_{\alpha \in A}$ has a convergent subnet. Indeed, if some subnet would tend to $+\infty$, then for every $c \in \mathbb{N}$ choose $\alpha$ such that $m_{\alpha}>c$; hence,

$$
\left(\mu_{m_{\alpha}}^{\alpha} x_{m_{\alpha}}+\mu_{m_{\alpha}+1}^{\alpha} x_{m_{\alpha}+1}+\cdots\right)_{\alpha \in B}
$$

converges to $x$, where $B$ is cofinal in $A$.

Considering the first $c$ components of this net, notice that they are

$$
s_{\alpha} q \oplus s_{\alpha} \varepsilon p_{1} \oplus \cdots \oplus s_{\alpha} \varepsilon p_{1} .
$$

Since $c \in \mathbb{N}$ was arbitrarily large, this implies that

$$
x=s q \oplus s \varepsilon p_{1} \oplus s \varepsilon p_{1} \oplus \cdots .
$$

Our assumption that $x \neq 0$ ensures $s \neq 0$, but this contradicts $x \in J(M)$.

The net $\left(m_{\alpha}\right)_{\alpha \in A}$ has a cluster point in $\overline{\mathbb{N}}$, the one-point compactification of $\mathbb{N}$. By the above remarks, this cluster point is different from $+\infty$.

Consequently, there exists an integer $n_{1} \geq 1$ and a subnet $\left(\lambda_{1}^{\alpha}\right)_{\alpha \in A_{1}}$ such that

$$
\left(\lambda_{1}^{\alpha} x_{n_{1}}+\mu_{n_{1}+1}^{\alpha} x_{n_{1}+1}+\cdots\right)_{\alpha \in A_{1}}
$$

converges to $x$. If we look at the $\left(n_{1}+1\right)$ st component of this net and multiply it by any nonzero projection $e \leq p_{1}-p_{2}$ we get $e\left(\mu_{n_{1}+1}^{\alpha}+\mu_{n_{1}+2}^{\alpha}+\cdots\right)$. Ultraweak convergence implies that

$$
s_{\alpha}-\lambda_{1}^{\alpha}=\mu_{n_{1}+1}^{\alpha}+\mu_{n_{1}+2}^{\alpha}+\cdots
$$

is convergent; hence, $\lambda_{1}^{\alpha}$ is a convergent net in $\mathbb{C}$. Suppose that $\lim _{\alpha} \lambda_{1}^{\alpha}=\lambda_{1} \in$ C. It follows that

$$
\lim _{\alpha}\left(\lambda_{1} x_{n_{1}}+\mu_{n_{1}+1}^{\alpha} x_{n_{1}+1}+\cdots\right)_{\alpha \in A_{1}}=x
$$

that is, $\lim _{\alpha}\left(\lambda_{1} x_{n_{1}}+y_{\alpha}^{1}\right)_{\alpha \in A_{1}}=x$, where $A_{1}$ is cofinal in $A$.

The construction continues by induction on $k$, after replacing $x$ by $x-$ $\lambda_{1} x_{n_{1}}-\cdots-\lambda_{k-1} x_{n_{k-1}}$. 
Let us prove the last assertion. Suppose that for some $\delta>0$ there is a sequence $\left(k_{i}\right)_{i \geq 1}$ such that $\left|s_{\alpha}^{k_{i}}\right| \geq \delta, \alpha \in A_{k_{i}}^{\prime}$ cofinal in $A_{k_{i}}$. For $0 \neq e \leq p_{1}-p_{2}$ define

$$
\begin{array}{ccc}
\tilde{\boldsymbol{e}}=0 \oplus \cdots \oplus 0 & \oplus \boldsymbol{e} \oplus 0 \oplus \cdots \oplus 0 & \oplus \boldsymbol{e} \oplus 0 \oplus \cdots . \\
\downarrow & \downarrow \\
n_{1}+1 & n_{2}+1
\end{array}
$$

Let us now remark that for every $\alpha \in A_{k_{j}}$ and for every $j \geq 1$ the first $n_{j}+1$ components of $\tilde{e} x_{\alpha}^{j}$ are

$$
\begin{array}{cccc}
0 \oplus \cdots \oplus 0 \oplus e s_{\alpha}^{1} \oplus 0 \oplus \cdots \oplus 0 & \oplus e s_{\alpha}^{2} \oplus 0 \oplus \cdots \oplus 0 & \oplus e s_{\alpha}^{j} \\
\downarrow & n_{2}+1 & n_{j}+1
\end{array}
$$

By taking into account our assumption that infinitely many $s_{\alpha}^{k_{i}}$ satisfy $\left|s_{\alpha}^{k_{i}}\right| \geq$ $\delta$, it follows that the operator $\tilde{e} x \in J(M)$ has infinitely many components of absolute value greater or equal to $\delta e$. This contradiction establishes our assertion and the fact that the series $\sum \lambda_{k}$ is convergent.

Notice that the operator $y_{\varepsilon}=q \oplus \varepsilon p_{1} \oplus \varepsilon p_{1} \oplus \varepsilon p_{1} \oplus \cdots$ belongs to $S_{\varepsilon}$.

Lemma 2.2. If $\varepsilon=m^{-2}(m \geq 6)$ and $y=y_{m^{-2}}$ then $\operatorname{dist}(y, J(M))=m^{-2}$ and $\operatorname{dist}\left(y, S_{m^{-2}} \cap J(M)\right) \geq m^{-1}$.

Proof. By removing the zeroth component, namely, the one corresponding to $q \in J(M)$, one can easily see that

$$
\operatorname{dist}(y, J(M))=\varepsilon=m^{-2} .
$$

Suppose that $\operatorname{dist}\left(y, S_{m^{-2}} \cap J(M)\right)<m^{-1}$, and choose $x \in S_{m^{-2}} \cap J(M)$ such that $\|y-x\|<m^{-1}$. With notation in Lemma 2.1 and its proof, it follows that $|1-s|<m^{-1}$. Choose $\alpha_{0}$ such that $\alpha \geq \alpha_{0}$ implies $\left|1-s_{\alpha}\right|<m^{-1}$. By Lemma 2.1, choose $k \geq 1$ such that $\left|s_{\alpha}^{k}\right|<m^{-1}, \alpha \in A_{k}^{\prime}$ cofinal in $A_{k}$, which entails $\left|1-\left(\lambda_{1}+\cdots+\lambda_{k}\right)\right|<2 m^{-1}$; hence, $\left|\lambda_{1}+\cdots+\lambda_{k}\right|>1-2 m^{-1}$. The $\left(n_{k}+1\right)$ st component multiplied by $p_{n_{k}+1}$ shows that $\|y-x\| \geq\left|\varepsilon-\left(\lambda_{1}+\cdots+\lambda_{k}\right)-\varepsilon s_{\alpha}^{k}\right|-m^{-1} \geq 1-3 m^{-1}-m^{-2}-m^{-3} \geq 1-5 m^{-1}$ for $\alpha$ large enough. Since the inequality $1-5 m^{-1}<m^{-1}$ fails to be true for $m \geq 6$, our assertion is proved.

Proposition 2.3. Every type $\mathrm{II}_{\infty}$ factor contains a local subspace whose compact ideal perturbation is not norm-closed.

Proof. If we define $S=\bigoplus_{m \geq 6} S_{m^{-2}}$, then $S$ is local in $M \otimes B\left(l^{2}(\mathbb{N})\right)$, but the canonical algebraic isomorphism between $(S+J(M)) / J(M)$ and $S /(S \cap J(M))$ fails to be a topological isomorphism, by Lemma 2.2. This proves that $S+J(M)$ is not norm-closed.

The general case is now immediate. Since $M=M_{0} \otimes B\left(l^{2}(\mathbb{N})\right)$, note that $M_{0} \otimes B\left(P_{m} l^{2}(\mathbb{N})\right)$ will contain a copy of $S_{m^{-2}}$, where $P_{m}$ is the projection of $l^{2}(\mathbb{N})$ onto the subspace generated by $\left\{\xi_{p_{m}}, \xi_{p_{m}^{2}}, \xi_{p_{m}^{3}}, \ldots\right\}, p_{m}$ being the $m$ th prime number. The projections $P_{m}$ are mutually orthogonal, which concludes the proof.

\section{ACKNOWLEDGMENT}

I would like to thank Professors V. Kaftal, D. R. Larson, and G. Weiss for stimulating conversations on the subject. 


\section{REFERENCES}

1. H. Bercovici and F. Pop, On perturbations of reflexive algebras, Rocky Mountain J. Math. 20 (1990), 379-387.

2. K. R. Davidson and S. C. Power, Best approximation in $C^{*}$-algebras, J. Reine Angew. Math. 368 (1986), 43-62.

3. J. Dixmier, Les $C^{*}$-algèbres et leur représentations, Gauthier-Villars, Paris, 1964.

4. T. Fall, W. Arveson, and P. Muhly, Perturbations of nest algebras, J. Operator Theory 1 (1979), 137-150.

5. J. Froelich, Compact operators, invariant subspaces and spectral synthesis, Ph.D. Thesis, University of Iowa, 1984.

6. F. Gilfeather, A. Hopenwasser, and D. R. Larson, Reflexive algebras with finite width lattices: Tensor products, cohomology, compact perturbations, J. Funct. Anal. 55 (1984), 176-199.

7. F. Gilfeather and D. R. Larson, Nest-subalgebras of von Neumann algebras, Adv. in Math. 46 (1982), 171-199.

8. V. Kaftal, D. R. Larson, and G. Weiss, Quasitriangular subalgebras of semifinite von Neumann algebras are closed, J. Funct. Anal. 107 (1992), 387-401.

9. C. Laurie, On density of compact operators in reflexive algebras, Indiana Univ. Math. J. 30 (1981), 1-16.

10. S. Popa and F. Radulescu, Derivations of von Neumann algebras into the compact ideal space of a semifinite algebra, Duke Math. J. 57 (1988), 485-518.

Department of Mathematics and Statistics, University of New Mexico, Albuquerque, New MeXICo 87131

E-mail address: florin@math.unm.edu

Current address: Department of Mathematics, Texas A\&M University, College Station, Texas 77843 